\title{
Situation Representation Model Implemented by Granule Fuzzy Characteristics in Mobile Autonomous System
}

Kargin Anatolii ${ }^{1}$

Luchentsov Yevhen ${ }^{2}$

\author{
${ }^{1}$ Ukrainian State University of Railway Transport, Feyerbakh \\ square, 7, Kharkov, 61050,kargin@kart.edu.ua \\ ${ }^{2}$ Ukrainian State University of Railway Transport, Feyerbakh \\ square, 7, Kharkov, 61050,luchentsov@kart.edu.ua
}

\begin{abstract}
Creation of the mobile autonomous control system has specifics. The decision-making and assessment of the large scale situation are based on the global situation model presented in the form of a multilevel structure at different abstraction levels. Three types of abstraction are considered: quantitative, deterministic, and generalized abstraction. The report examines the algorithm for processing data from sensors at the first two levels. Algorithm is programmed in Python and implemented on ESP 8266 microcontroller.
\end{abstract}

Keywords: mobile autonomous systems, global situation model, quantitative and determinative abstraction, granule fuzzy characteristics.

\section{INTRODUCTION AND PROBLEM STATEMENT}

Large scale situation assessment systems, organized on a distributed monitoring basis, include local components that move across the monitoring object space and are implemented as mobile autonomous systems (MAS). The decision-making and assessment of the situation in these systems are based on the global situation model (GSM) [1], which is based on data obtained from MAS. In work [2], GSM is proposed in the form of a multilevel structure at different levels, the situation of which is represented by concepts of different levels of abstraction. Model [3] formalized three types of data abstraction: quantitative, deterministic, and generalized abstraction. Quantitative and determinative abstraction (Q\&DA) acts as a bridge between natural language description and numerical sensor data. The report examines the first two levels of abstraction of Q\&DA for the representation of GSM in MAS.

At the department of Information Technologies of USURT, the applications creation technologies, including MAS, are tested on the basis of a training and research polygon of the Internet of Things and Intelligent Machines [1]. In the report, a wheeled robot performing the function of monitoring the occurrence of a fire situation is views as a MAC. The robot is implemented on a four-wheeled chassis with gear motors, equipped with a single-board Raspberri Pi 3B computer, Arduino Motor Shield controller and ESP 8266 microcontroller. Data acquisition from DHT11 temperature and humidity sensors, BH1750 smoke, MQ - 2 flame and illumination and Q\&DA is implemented on ESP 8266 microcontroller. The report examines the algorithm for processing data from sensors at the first two levels of Q\&DA. Algorithm is programmed in Python and implemented on ESP 8266 microcontroller.

\section{PROBLEM SOLUTION AND RESULTS}

The algorithm uses knowledge of granulation of a control variable: how many granules are by the range of possible variable values) and fuzzy restrictions for these granules. The fuzzy characteristic of the pellets is the fuzzy confidence factor $[2,3]$. Therefore, the fuzzy constraints of the granules are set as a function of the fuzzy confidence factor given in the variable definition area, as shown in Fig. 1.

Q\&DA is a procedure of granulating data from each source and determining granules fuzzy characteristics (GFC) [3]. For this, set of possible sensor values covered by several information pellets. The size and number of granules depends on the task. Each of these granules refers to the zero level of GSM. The fuzzy characteristic of the pellets is a fuzzy L-R number with a Gaussian function with three parameters: $\alpha$ confidence; TL and TR are the time intervals since the last sensor data was received and the data changed, respectively. On the basis of GFC there is an integral characteristic of confidence - a confidence factor CF [3].

The universal model for presenting knowledge about granulating data from a single sensor is as follows:

$$
\begin{gathered}
<n,\left(a_{1}, b_{1}, c_{1}, d_{1}, e_{1}, f_{l}\right), \ldots,\left(a_{i}, b_{i}, c_{i}, d_{1}, e_{i}, f_{i}\right), \ldots, \\
\left(a_{n}, b_{n}, c_{n}, d_{n}, e_{n}, f_{n}\right)>,
\end{gathered}
$$

where $n-$ is the number of granules into which the area of possible data from sensor is broken;

$a_{i}, b_{i}, c_{i}, d_{l}, e_{i}, f_{i}$ - are parameters of a piecewise linear function representing the distribution of confidence $\alpha$ over the range of possible data from sensor.

The meaning of the parameters $a_{i}, b_{i}, c_{i}, d_{l}, e_{i}, f_{i}$ explains Fig. 1b. Consider an example where the data from the temperature sensor is represented by 3 granules. Formula (1) for this case will be:

$$
\begin{gathered}
<3,\left(a_{1}=20, b_{1}=20, c_{1}=20, d_{1}=24, e_{1}=28, f_{1}=38\right), \\
\left(a_{2}=20, b_{2}=24, c_{2}=28, d_{2}=32, e_{2}=36, f_{2}=38\right), \\
\left(a_{3}=20, b_{3}=32, c_{3}=36, d_{3}=38, e_{3}=38, f_{3}=38\right)>
\end{gathered}
$$

The numerical definition of the three granules in (2) is shown graphically in Fig. 1 and corresponds: the first granule is shown in Fig. 1a, the second is shown in Fig. 1b, and the third is shown in Fig. 1.c. 


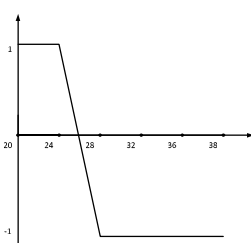

a)

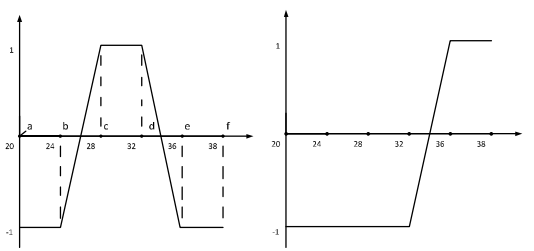

b) c)
Figure 1. Graphical view of the numerical definition

In accordance with the abstraction model [2,3], an algorithm for granulating the input numerical values and extracting GFC in real time was developed. The granulation algorithm for a single data processing step on the example of a single temperature sensor is shown below.

1. Obtaining data from the temperature sensor $\mathrm{T}$.

2. Validation of data $T \in\left[T_{\min }, T_{\max }\right]$.

3. Calculation of the confidence parameter $\alpha$.

$$
\alpha= \begin{cases}-1 & \text { if } T \in(a, b], b \neq T_{\min } \text { or } T \in(e, f] \\ -1+2 \frac{T-b}{c-b} & \text { if } T \in(b, c], c \neq T_{\min } ; \\ +1-2 \frac{T-d}{e-d} & \text { if } T \in(d, e] \\ +1 & \text { if } T \in(\mathrm{c}, \mathrm{d}] .\end{cases}
$$

4. Calculation of confidence parameters $t_{L}$ and $t_{R}$

$$
t_{L}=0
$$

$t_{R}= \begin{cases}0, & \text { if }(\alpha \geq \varepsilon \quad \&-q=0) \text { or }\left(\alpha \leq-\varepsilon \quad \&{ }^{-} q=1\right) \\ -t_{R}+1, & \text { therwise, }\end{cases}$

\section{CONCLUSION}

The following example demonstrates the efficiency of a program. The results of a survey of the temperature sensor at the current step gave $\mathrm{T}=27^{\circ}$. Assuming that in the previous step were the values of $G F C_{1}=\left(\alpha_{1}=-1.0, t_{L 1}=0, t_{R 1}=10\right)$, $G F C_{2}=\left(\alpha_{2}=-1.0, t_{L 2}=0, t_{R 2}=10\right), G F C_{3}=\left(\alpha_{3}=0.75, t_{L 3}\right.$ $\left.=0, t_{R 3}=10\right)$ and $\varepsilon=0.75$. Calculations according to the above algorithm based on knowledge (2) gave the following values of the parameters of GFC for these granules: $G F C_{I}=$ $\left(\alpha_{1}=-0,5, t_{L 1}=0, t_{R 1}=11\right), G F C_{2}=\left(\alpha_{2}=0.5, t_{L 2}=0, t_{R 2}=\right.$ 11), $G F C_{3}=\left(\alpha 3=-1, t_{L 3}=0, t_{R 3}=0\right)$.

\section{REFERENCES}

[1] A. Kargin,. O. Ivaniuk, G. Galych, A. Panchenko, "Pollygon for smart machine application", 2018 IEEE 9th International Conference on Dependable Systems, Services and Technologies DESSERT'2018, Ukraine, Kyiv, May 24-27, 2018, P. 489-494.

[2] A. Kargin, T. Petrenko, "Abstraction and categorization in smart machines based on granular computations." Vestnik Natsional'nogo tekhnicheskogo universiteta" KhPI". Seriya: Informatika i modelirovaniye, vol. 50(1271), pp. 57-68, 2017. (in Russian)

[3] A. Kargin, T. Petrenko, "Internet of Things Smart Rules Engine", in 2018 Inter. Sci.-Pract. Conf. Probl. Infocommun. Sci. and Technol. (PIC S\&T 2018), Kharkiv, Ukraine, Oct. 9-12, 2018, pp. 639-644. 